\title{
Artery Cell Contraction via ROS and NO Balance Examined by In Vivo Multi-photon Imaging Technique and Laser Injuries Technique
}

\author{
Satoshi Nishimura ${ }^{1,2,3}$
}

1Department of Cardiovascular Medicine, the University of Tokyo, Hongo 7-3-1, Bunkyo-ku, Tokyo, Japan

2Translational Systems Biology and Medicine Initiative, the University of Tokyo, Hongo 7-3-1, Bunkyo-ku, Tokyo, Japan

4Jichi Medical University, Tochigi, Japan

Blood pressure is regulated dynamically by arterial wall tensions in vivo, but the previous methods did not clarify their detailed mechanisms in living animals due to the lack of visualization technique. Thus we aimed to visualize cell kinetics during artery contractions processes by combining in vivo multi-photon imaging and photochemical reaction technique by laser injuries.

We recently developed the in vivo fluorescent imaging technique based on two photon microscopy, which enabled us to directly visualize the femoral artery in living animals. In addition, by combining with the visualization technique with laser induced injury models, we could visualize transient artery contraction in response to ROS (reactive oxygen species) (Figure a). Using this novel animal model, we elucidated the functional contribution of smooth muscle cell of artery to hypertensive diseases. We also directly measured the ROS and NO (nitric oxide) production in smooth muscle cells, which was analyzed and quantified by novel tracking software.

We observed that ROS and NO production was counter-balanced in transient artery contractions (Figure $\mathrm{b}$ and $\mathrm{c}$ ). As for the molecular mechanisms, we confirmed that this contraction is via PKC and NADPH signaling. The contraction was inhibited by $\mathrm{Ca}$ blocker, isosorbide, sildenafile, and angiotension II blockers administrations.

In addition, by treating the artery with $\mathrm{CaCl}$ solution, the aneurysm formation was also visualized. In the early phase, the hyper-responsiveness of artery wall, and transient thrombus formation was observed. In the later, the wall was stiffened, collagen contents were increased, and aneurysm was observed in last.

These results indicated the ROS and NO counter balances dynamically regulate artery contractions. eNOS also contributed to these contractions, and imaging can visualize various dynamic signaling. .In sum, our imaging system can be a powerful tool to analyze the molecular mechanisms of hypertension and artery aneurysm.

\section{References:}

[1] Nishimura et al, 2012 Blood.

[2] Nishimura et al, $2010 \mathrm{JCI}$.

[3] Nishimura et al, 2009 Nat Med.

[4] Nishimura et al, 2013 Cell Metabolism

[5] Nishimura et al, 2008 JCI. 

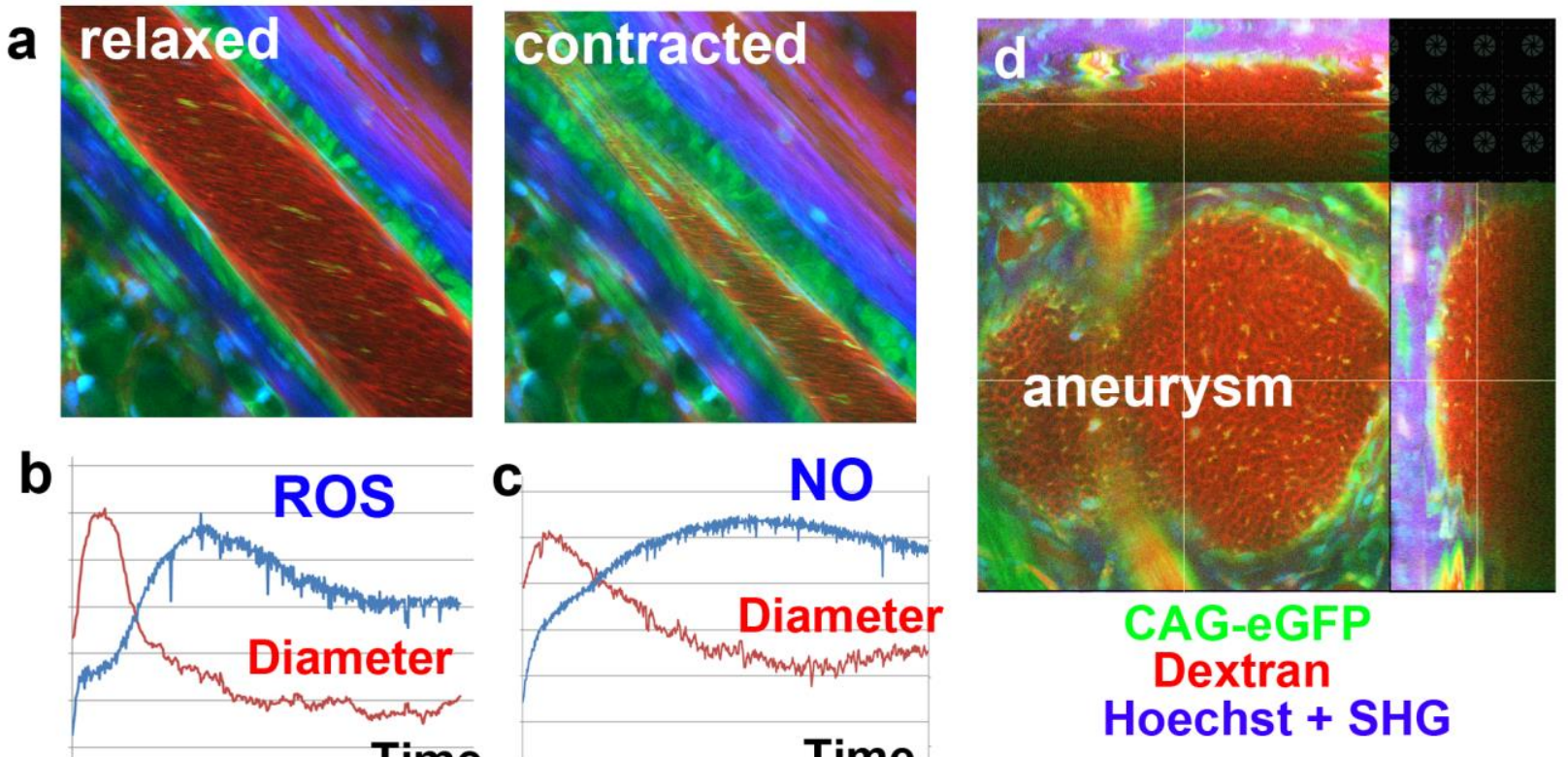

Time

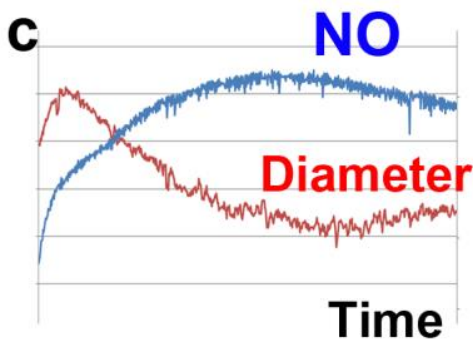

Figure : In vivo imaging of femoral arteries and contractions by ROS stimulation (Green: CAG-eGFP, Red: dextran, Blue: Hoechst) 